\title{
Long Vascular Sheaths for Transfemoral Neuroendovascular Procedures in Children
}

\author{
Adam A. Dmytriw, MD, MPH, MSc${ }^{1}$, Winston Ha, MD $^{1}$, Suzanne Bickford ${ }^{1}$, Kartik Bhatia, MD ${ }^{1}$, \\ Manohar Shroff, $\mathrm{MD}^{1}$, Peter Dirks, $\mathrm{MD}^{2}$, Prakash Muthusami, $\mathrm{MD}^{1}$ \\ ${ }^{1}$ Neuroradiology and Image Guided Therapy, Hospital for Sick Children, Toronto, ON, Canada \\ ${ }^{2}$ Department of Neurosurgery, Hospital for Sick Children, Toronto, ON, Canada
}

Purpose: To evaluate the safety and efficacy of long vascular sheaths for transfemoral neuroendovascular procedures in children.

Materials and Methods: A retrospective evaluation of transfemoral neuroendovascular procedures in children $<18$ years, using long sheaths was undertaken analyzing procedure type, fluoroscopic times, technical success, access site and systemic complications. Twenty-seven consecutive procedures were included over a 2-year period. Mean age was 8.4 years (standard deviation [SD] 6.3) (range 17.0 months-16.3 years).

Results: Patients were 44\% female and mean weight was $35.0 \mathrm{~kg}$ (SD 22.8) (range 9.8-72.2 kg). A third of the procedures were performed in $\leq 15 \mathrm{~kg}$ children. The most common procedure was for embolization ( $n=13,48.1 \%)$ and the most common indication was dual microcatheter technique (52\%). The most common device used was the $5 \mathrm{Fr}$ Cook Shuttle sheath. Mean fluoroscopy time was 61.9 minutes (SD 43.1). Of these procedures, $93 \%$ were technically successful. Femoral vasospasm, when present, was self-limiting. Complications (3/27, 11.1\%) included groin hematoma $(n=1)$, neck vessel spasm that resolved with verapamil $(n=1)$, and intracranial thromboembolism ( $n=1)$, with no significant difference between the $\leq 15 \mathrm{~kg}$ and $>15 \mathrm{~kg}$ subcohorts. There were no aorto-femoro-iliac or limb-ischemic complications.

Conclusion: Long vascular sheaths without short femoral sheaths can be safely used for pediatric neuroendovascular procedures as they effectively increase inner diameter access without increasing the outer sheath diameter. This property increases the range of devices used and intracranial techniques that can be safely performed without arterial compromise, thus increasing the repertoire of the neurointerventionist.

Key Words: Angiography; Catheter; Pediatrics; Feasibility study; Radiology

\section{INTRODUCTION}

Long arterial sheaths are commonly used in neurovascular interventions in adults for providing increased support and the ability to deliver multiple intracranial devices. ${ }^{1,2}$ Long sheaths have traditionally been avoided in pediatric neu- roendovascular practice, and more so in infants and small children. In each case, the added benefits must be weighed against the larger caliber size and stiffness of these sheaths. A number of potential advantages are inherent with long sheath use which could outweigh concerns if employed safely, including

\section{Correspondence to:}

Adam A. Dmytriw, MD, MPH, MSc Neuroradiology and Image Guided Therapy, Hospital for Sick Children, 555 University Avenue, Toronto, ON M5G 1X8, Canada

Tel: $+1-416-813-5900$

Fax: +1-416-813-5901

E-mail:adam.dmytriw@sickkids.ca

Received: April 7, 2021

Revised: May 14, 2021

Accepted: May 21, 2021
Copyright $\odot 2021$ Korean Society of Interventional Neuroradiology

This is an Open Access article distributed under the terms of the Creative Commons Attribution Non-Commercial License (http://creativecommons.org/licenses/by-nc/4.0) which permits unrestricted non-commercial use, distribution, and reproduction in any medium, provided the original work is properly cited. 
the ability to co-utilize intermediate access catheters which itself affords improved technical nuances and thus safety, and potentially even shorter procedure times. In addition, dual microcatheter and microballoon techniques could be used, which would otherwise require bilateral access. Bilateral access imposes its own set of risks, not least the increased potential for loss of limb in small children. Procedural plans are devised to achieve the best possible results with available access, at times leading to a compromise in technique.

Risks entertained from using a long sheath include target vessel vasospasm or dissection from navigating a largebore device in smaller and more reactive pediatric arteries, as well as potential occlusion and its long-term neurological sequelae. Furthermore, a long sheath employed in isolation without a shorter sheath could imply prolonged occlusion at the access site, which may be particularly undesirable during pediatric intervention. Transfemoral long sheath use was recently shown in the cardiology literature to result in a nearly $40 \%$ incidence of femoral arterial complications in infants $\leq 15 \mathrm{~kg}^{3}$. There are no corresponding data in the neurointer-

\section{Table 1. Our protocol for long sheath use in pediatric neuroendovascular procedures}

Patient preparation and procedure planning

- Image review for procedure plan

- Focused ultrasound of both femoral arteries by interventionist to assess size (for 5 Fr long sheath which has outer diameter of 7 Fr, a minimum diameter of 3.0-mm is required), and anatomical variants (e.g., high bifurcation of common femoral artery)

- Decision making regarding short sheath vs. long sheath vs. bilateral access based on above plan and ultrasound findings

- Marking of pedal pulses and pulse oximeter attached to toe(s) on side(s) of access

Access

- Ultrasound guidance for access. Highest frequency linear transducer that can adequately show femoral artery, with focus adjusted to required depth. Most commonly used is the 15-7 $\mathrm{MHz}$ linear transducer.

- Micropuncture set for access. Out of plane technique used to confirm entry in the mid-point of arterial wall. Only single wall puncture used

- No tract dilatation used unless necessary

- Pharmacological vasodilators not used routinely. In case of femoral vasospasm reflected by sustained reduction of distal $\mathrm{SpO}_{2}<80 \%$, nitroglycerin $(3 \mu \mathrm{g} / \mathrm{kg}$, maximum of $200 \mu \mathrm{g})$ injected slowly over 1-minute through micropuncture cannula

- If direct insertion of long sheath, ensure no/minimal transition between sheath and inner dilator/catheter. If short sheath already in place, exchanged with external carotid access. Fixed core 0.035 inch wire used in both cases with a floppy tip formed into a J-shape

Intra-procedural

- Pulse oximeter maintained on ipsilateral foot throughout procedure

- Heparin given as bolus, 75-100 U/kg

- Tip of sheath positioned in a straight vessel segment, preferably below common carotid bifurcation for internal/external carotid artery procedures, and in the subclavian artery but not beyond the V1-segment if advanced into a non-tortuous left vertebral artery for posterior circulation procedures

- Check contrast injection to confirm no vasospasm, vessel wall injury or contrast stagnation

- Once in desired position, sheath secured with tape to anterior thigh to minimize translational motion

- Sheath maintained on continuous flush with heparinized saline

- In the event of sheath-related neck vessel spasm during insertion or anytime during the procedure, intra-arterial verapamil (0.5 mg/mL; 1-mL in infants and 2-mL in small children $<30 \mathrm{~kg}$, over 1-minute) administered through the sheath. If severe spasm, sheath withdrawn into brachiocephalic/aorta and check performed in 5-10 minutes

Post-procedural

- Hemostasis by manual compression or closure device, at the discretion of the operator. In case of closure device, perform check femoral angiography

- Check ultrasound and Doppler examination of the access site by the interventionist following hemostasis, to confirm arterial patency and flow into limb

- No pressure dressing applied routinely unless difficult hemostasis. Visual inspection of arterial access site for bleeding, hematoma, bruise by bedside nurse

- Pedal pulses monitored by nurse (q15 min×4, q30 min×4). Limb monitored for color and capillary refill

- For infants or in case pedal pulse non-palpable, pulse oximeter maintained on foot for 3 hours post-hemostasis; for older children only if access-site complications e.g., vasospasm encountered

- Patient can ambulate after 6 hours of hemostasis

- Post-discharge care: can perform routine daily activities, no sports or gym for 2 weeks. In case of active bleeding or sudden swelling, to apply pressure and return to the emergency department

- Clinical assessment of access site and distal limb including pulses at the next clinic visit; in case of concern with swelling, thrill or reduced distal perfusion, ultrasound and Doppler examination performed 
ventional literature.

We hypothesized that in a high volume pediatric cerebrovascular center, using dedicated protocols, the use of long sheaths for transfemoral neuroendovascular procedures can lend technical nuance without significant device-related morbidity. The goal of this study was thus to evaluate the safety and efficacy of using long vascular sheaths for neurointerventional procedures in children. A secondary objective was to look in particular at the risk profile for children $\leq 15 \mathrm{~kg}$, who from the cardiology literature had significant complication rates with the use of long transfemoral arterial sheaths.

\section{MATERIALS AND METHODS}

An Institutional Review Board-approved retrospective evaluation was performed of transfemoral neuroendovascular procedures in children $<18$ years, performed using a dedicated protocol (Table 1) in our institute over a 2-year period from 2016-2018, using long sheaths or guide catheters without an intervening short sheath. As guide catheters do not typically use a dilator, guide catheters without short sheaths were introduced over a diagnostic catheter. Long sheaths and guide catheters used were the Flexor Shuttle Sheath (5 Fr outer diameter [OD], 0.074 inch inner diameter [ID]), the

Table 2. Baseline characteristics of patients in our cohort ( $n=23$ patients, 27 procedures)

\begin{tabular}{lccc}
\hline Variable & All patients $(n=23)$ & $\leq 15 \mathrm{~kg}$ patients $(\mathrm{n}=9)$ & $>15 \mathrm{~kg}$ patients $(\mathrm{n}=18)$ \\
\hline Mean age $(\mathrm{y})$ & $8.4 \pm 6.3$ & $1.7 \pm 0.5$ & $11.7 \pm 4.9$ \\
Mean weight $(\mathrm{kg})$ & $35.0 \pm 22.8$ & $11.7 \pm 1.8$ & $46.7 \pm 19.1$ \\
Sex, male/female & $15 / 12$ & $4 / 5$ & $11 / 7$ \\
Diagnosis & & & \\
Retinoblastoma & $10(37.0)$ & $1(11.1)$ & $2(11.1)$ \\
Brain arteriovenous malformation & $8(29.6)$ & $0(0)$ & $7(38.9)$ \\
Arterial ischemic stroke & $3(11.1)$ & $0(0)$ & $3(16.7)$ \\
Brain aneurysm & $2(7.4)$ & $0(0)$ & $2(11.1)$ \\
Tumor & $2(7.4)$ & $0(0)$ & $2(11.1)$ \\
Traumatic pseudoaneurysm & $1(3.7)$ & $0(0)$ & $1(5.6)$ \\
Epistaxis & $1(3.7)$ & $1(5.6)$ & \\
\hline
\end{tabular}

Values are presented as mean \pm standard deviation or number (\%).
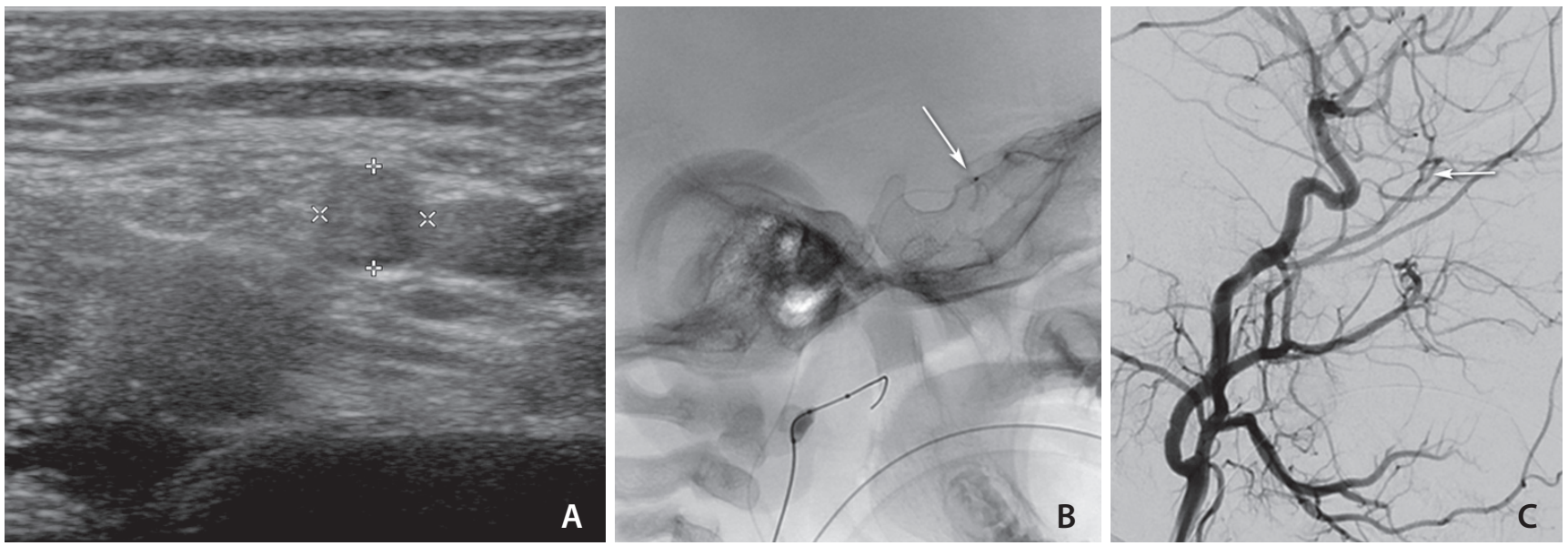

Fig. 1. Dual microcatheter via single transfemoral long sheath. Ten-month female with retinoblastoma. (A) Initial ultrasound performed to confirm adequate size of the common femoral artery. The artery measured $4.1 \times 3.9 \mathrm{~mm}$. (B) Hemodynamic modulation performed by balloon occlusion across the ostium (arrow) of the middle meningeal artery, in order to permit antegrade ophthalmic arterial flow. (C) Common carotid injection showing dual supply to the usually located ophthalmic artery. Arrow pointing to the tip of the microcatheter in the ophthalmic arterial ostium. 
Table 3. Procedural data for neurointerventional surgeries in our cohort ( $n=23$ patients, 27 procedures)

\begin{tabular}{|c|c|c|c|}
\hline Variable & $\begin{array}{l}\text { All procedures } \\
(\mathrm{n}=27)\end{array}$ & $\begin{array}{l}\leq 15 \mathrm{~kg} \text { procedures } \\
(\mathrm{n}=9)\end{array}$ & $\begin{array}{l}>15 \mathrm{~kg} \text { procedures } \\
(\mathrm{n}=18)\end{array}$ \\
\hline \multicolumn{4}{|l|}{ Reason for long sheath } \\
\hline Dual microcatheter technique & $14(51.9)$ & $6(66.7)$ & $8(44.4)$ \\
\hline Triaxial support & $9(33.3)$ & $3(33.3)$ & $6(33.3)$ \\
\hline Flow reversal & $3(11.1)$ & $0(0)$ & $3(16.7)$ \\
\hline Intra-operative angiography & $1(3.7)$ & $0(0)$ & $1(5.6)$ \\
\hline \multicolumn{4}{|l|}{ Procedure } \\
\hline Embolization & $13(48.1)$ & $1(11.1)$ & $12(66.7)$ \\
\hline Embolization of shunting lesions & $9(33.3)$ & $1(11.1)$ & $8(44.4)$ \\
\hline Tumor embolization & $2(7.4)$ & $0(0)$ & $2(11.1)$ \\
\hline Aneurysm treatment & $1(3.7)$ & $0(0)$ & $1(5.6)$ \\
\hline Pseudoaneurysm embolization & $1(3.7)$ & $0(0)$ & $1(5.6)$ \\
\hline Intra-arterial chemotherapy & $10(37.0)$ & $8(88.9)$ & $2(11.1)$ \\
\hline Mechanical thrombectomy for stroke & $3(11.1)$ & $0(0)$ & $3(16.7)$ \\
\hline Diagnostic angiography (intra-operative) & $1(3.7)$ & $0(0)$ & $1(5.6)$ \\
\hline \multicolumn{4}{|l|}{ Sheath/Catheter used without short sheath } \\
\hline 5 Fr Flexor Shuttle sheath (ID 0.074 inch) & $16(59.3)$ & $8(88.9)$ & $8(44.4)$ \\
\hline $\begin{array}{l}\text { Penumbra Benchmark intracranial access catheter } \\
\text { (OD } 6 \text { Fr, ID } 0.071 \text { inch)* }\end{array}$ & $5(18.5)$ & $0(0)$ & $5(27.8)$ \\
\hline 6 Fr Penumbra Neuron Max sheath (ID 0.088 inch) & $4(14.8)$ & $0(0)$ & $4(22.2)$ \\
\hline 6 Fr Flexor Shuttle sheath (ID 0.087 inch) & $1(3.7)$ & $1(11.1)$ & $0(0)$ \\
\hline $\begin{array}{l}\text { Microvention Chaperon guiding catheter } \\
\text { (OD } 6 \text { Fr, ID } 0.071 \text { inch) }\end{array}$ & $1(3.7)$ & $0(0)$ & $1(5.6)$ \\
\hline \multicolumn{4}{|l|}{ Vessel accessed } \\
\hline Internal carotid artery & $10(37.0)$ & $7(77.8)$ & $3(16.7)$ \\
\hline Common carotid artery & $9(33.3)$ & $0(0)$ & $9(50.0)$ \\
\hline Brachiocephalic & $2(7.4)$ & $1(11.1)$ & $1(5.6)$ \\
\hline Left vertebral artery & $2(7.4)$ & $0(0)$ & $2(11.1)$ \\
\hline Right Subclavian Artery & $2(7.4)$ & $0(0)$ & $2(11.1)$ \\
\hline Aorta & $1(3.7)$ & $0(0)$ & $1(5.6)$ \\
\hline Left Subclavian & $1(3.7)$ & $1(11.1)$ & $0(0)$ \\
\hline \multicolumn{4}{|l|}{ Heparin use } \\
\hline Yes & $25(92.6)$ & $9(100)$ & $16(88.9)$ \\
\hline Mean procedural heparin dose (U/kg) & $101.7 \pm 38.0$ & $98.7 \pm 4.0$ & $103.3 \pm 47.9$ \\
\hline Ultrasound guidance for access & $27(100)$ & $9(100)$ & $18(100)$ \\
\hline Mean fluoroscopy time (min) & $62.0 \pm 43.1$ & $45.8 \pm 39.1$ & $70.6 \pm 43.7$ \\
\hline Mean procedure time (h) & $4.5 \pm 2.2$ & $4.0 \pm 1.2$ & $4.9 \pm 2.8$ \\
\hline \multicolumn{4}{|l|}{ Post-procedural hemostasis } \\
\hline Manual pressure & 25 (92.6) & $8(88.9)$ & $17(94.4)$ \\
\hline Closure device & $2(7.4)$ & $1(11.1)$ & $1(55.6)$ \\
\hline
\end{tabular}

Values are presented as number (\%) or mean \pm standard deviation.

OD, outer diameter; ID, inner diameter.

*Employed as sheath. 
Penumbra Benchmark intracranial access catheter (6 Fr OD, 0.071 inch ID), the Penumbra Neuron Max sheath (6 Fr OD, 0.088 inch ID), the Flexor Shuttle sheath (6 Fr OD, 0.087 inch ID), and the Microvention Chaperon guiding catheter (6 Fr OD, 0.071 inch ID).

A total of 27 consecutive procedures in 23 children were included, where long sheaths or guide catheters were used for intracranial access without an intervening short femoral sheath. The mean age at time of procedure was $8.4 \pm 6.3$ years, range 17.0 months-16.3 years, 44\% female (Table 2). The mean weight of the children at time of procedure was $35.0 \pm 22.8 \mathrm{~kg}$, range $9.8-72.2 \mathrm{~kg}$. Non-neurovascular procedures were excluded. Procedural and imaging details obtained from electronic patient records and radiology picture archiving and communication system (PACS). Baseline patient characteristics were recorded, with mean age, weight, heparin dose, fluoroscopy time, and procedure time reported with standard deviations. Sex, diagnosis, procedure, reason for long sheath, procedure performed, sheath/catheter used, vessel accessed, heparin use, mean heparin dose, ultrasound guidance, complications, procedure efficacy, and post-procedural hemostasis were reported with counts and column percentages.

Values were reported as mean and standard deviation. Non-parametric chi-square test was used to compare complication rates between the two groups. P-value $<0.05$ was considered statistically significant. Data analysis was performed using GraphPad (GraphPad Software Inc., San Diego, (A, USA)

\section{RESULTS}

\section{Baseline patient characteristics and procedural data}

The most common procedure was for embolization $(n=13$, 48.1\%) and the most common indication for larger access ( $n=14,52 \%)$ was dual microcatheter technique (Fig. 1A-C). Long sheaths/catheters were used in the carotid artery (ICA or CCA) in $70 \%$ of all procedures, and $7.4 \%$ in the vertebral artery. The most frequent device used was the $5 \mathrm{Fr}$ Cook Shuttle sheath $(n=16,59 \%)$. Ultrasound guidance was used in all cases with micropuncture technique and single-wall puncture for femoral arterial access. Systemic heparin was used in all but 2 cases (ruptured arteriovenous malformation [AVM], iatrogenic pseudoaneurysm). Hemostasis was achieved either by manual compression (93\%) or Angioseal closure device (7\%) (Terumo Medical, Somerset, NJ, USA) if activated clotting time was two times the pre-procedure baseline. Mean fluoroscopy time was $61.9 \pm 43.1$ minutes (Table 3).

\section{Primary endpoint-safety and efficacy}

25/27 (93\%) of the procedures were technically successful (Table 4). The two technically unsuccessful procedures were intra-arterial chemotherapy sessions for retinoblastoma, where the ophthalmic artery or alternate route could not be accessed. Femoral vasospasm, when present, was self-limiting without requiring pharmacological vasodilatation in any case. There were no instances of femoro-ilio-aortic dissections or occlusions, no femoral pseudoaneuryrsms and no distal extremity ischemic complications, either at discharge or at clinical follow-up.

\section{Secondary endpoint for patients $\leq \mathbf{1 5} \mathbf{~ k g}(\mathbf{n}=\mathbf{9})$}

In the 9 cases below $15 \mathrm{~kg}$, the mean fluoroscopy time was $45.8 \pm 39.1$ minutes, and there were two complications (22.2\%); a groin hematoma (self-limiting) and an intracranial thromboembolic complication (asymptomatic distal emboli in a 2 year old, secondary to microcatheter-related focal internal carotid dissection). In the 18 procedures in children above $15 \mathrm{~kg}$,

Table 4. Procedural complications in our cohort ( $n=27$ procedures)

\begin{tabular}{|c|c|c|}
\hline Variable & Number (\%) & Mean weight $(\mathrm{kg})$ \\
\hline \multicolumn{3}{|l|}{ Complications } \\
\hline None & $24(88.9)$ & 36.6 \\
\hline $\begin{array}{l}\text { Groin swelling (post- } \\
\text { procedure) }\end{array}$ & $1(3.7)$ & 10.3 \\
\hline $\begin{array}{l}\text { Thromboembolic } \\
\text { complication }\end{array}$ & $1(3.7)$ & 12.5 \\
\hline $\begin{array}{l}\text { Vasospasm requiring } \\
\text { intra-arterial vasodilator }\end{array}$ & $1(3.7)$ & 45.4 \\
\hline \multicolumn{3}{|l|}{ Efficacy } \\
\hline Successful & 25 (92.6) & 34.2 \\
\hline Unsuccessful & $2(7.4)$ & 44.7 \\
\hline \multicolumn{3}{|l|}{ Efficacy for $\leq 15 \mathrm{~kg}$ patients } \\
\hline Successful & $8(88.9)$ & 11.6 \\
\hline Unsuccessful & $1(11.1)$ & 12.5 \\
\hline \multicolumn{3}{|l|}{ Efficacy for $>15 \mathrm{~kg}$ patients } \\
\hline Successful & $17(94.4)$ & 48.4 \\
\hline Unsuccessful & $1(5.6)$ & 16.9 \\
\hline
\end{tabular}


the mean fluoroscopy time was $70.6 \pm 43.7$ minutes, and there was one patient (5.6\%) with sheath insertion-induced vasospasm requiring and responsive to intra-arterial pharmacological vasodilation. Chi-squared test showed no significant difference $(P=0.20)$ in complication rates between these two groups.

\section{DISCUSSION}

The use of long femoral sheaths in the pediatric population for neuroendovascular procedures has the potential to enable advanced techniques which would improve patient care, should the risks involved be low. At our institution, long sheath use enabled dual microcatheter techniques, microballoon assisted procedures, triaxial support (Fig. 2A, B), flow-reversal for mechanical thrombectomy, and intraoperative angiography with an acceptable safety and efficacy profile for these complex procedures in children. In the vast majority (59.3\%) of cases, a 5 French Shuttle (Cook Medical, Bloomington, IN, USA) was used, with the various $6 \mathrm{Fr}$ sheaths or guide catheters comprising the remainder. Access
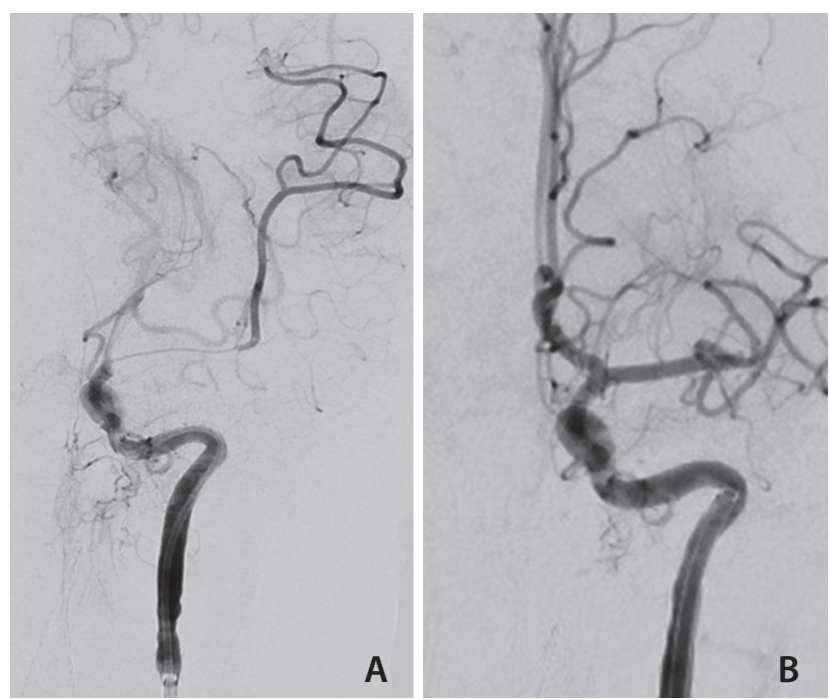

Fig. 2. Triaxial support for mechanical thrombectomy in a 12 -year old girl. (A) Long sheath (Neuron Max, 6 Fr, 0.088 inch inner diameter [ID]) with tip in the internal carotid artery, permits use of intermediate catheter (Sofia Distal Access Catheter, 5 Fr, 0.055 inch ID), while also permitting aspiration during thrombectomy. A complete occlusion by clot of the terminal internal carotid artery is seen, extending into the post-bifurcation middle cerebral artery (B) Angiogram following first passage of stent retriever (Trevo 4×30 mm), with complete large vessel recanalization. A second pass was subsequently performed for post-bifurcation-middle cerebral artery (MCA) clot. devices were most commonly positioned either in the cervical ICA (37.0\%) or CCA (33.3\%).

The proceduralist's discretion to attempt long sheath access was typically done in the setting of angiography and embolization of arteriovenous malformations/fistulae, tumors, for intra-arterial chemotherapy or mechanical thrombectomy for stroke. Arteriovenous intracranial shunting lesions are challenging lesions, mandating careful technical nuance that is made easier by dual microcatheter technique to enable optimal nidal penetration while avoiding nontarget embolization and reflux of liquid embolic agent. Several recent technical advancements, predominantly described in the adult literature, including microballoon assistance, ${ }^{4}$ use of newer liquid embolic agents like Onyx and precipitating hydrophobic injectable liquid (PHIL), 5,6 and pressure cooker technique, ${ }^{7}$ all of which have been shown to increase the efficacy and safety of endovascular treatment, have a limited role with a single $4 \mathrm{Fr}$ access that is traditionally employed in children. ${ }^{8}$ Similarly, treatment of pial arteriovenous fistula (AVF) and fistulous points of nidal AVMs have been thus far dependent on carefully controlled injections of n-butyl cyanoacrylate, ${ }^{9-12}$ which although effective, have a significant risk profile. ${ }^{12}$ Using long sheaths in these cases enables precise embolization via the use of microballoons, 13,14 or proximal coiling ${ }^{15,16}$ prior to liquid embolic injection, without the need for second femoral arterial access. This not only increases the efficacy of shunt closure, but enables a degree of technical control which enhances procedural safety. In the case of intra-arterial chemotherapy, careful navigation to the ophthalmic artery is required, for targeted drug delivery. In our cohort, long sheaths were used in select cases that had dual ophthalmic arterial supply, with a prominent meningo-ophthalmic collateral resulting in competitive flow that prevented adequate antegrade perfusion in the ophthalmic artery. In these cases, long sheaths allowed the use of microballoon across the middle meningeal artery ostium to alter the local hemodynamics to maximize flow and thereby drug delivery toward the central retinal and ciliary arteries. In other cases, microballoons were used to improve microcatheter stability at the ophthalmic arterial ostium when access or distal purchase was not feasible due to angioanatomy. Improved stability of an endovascular system for the purposes of intracranial aneurysm or tumor embolization are also well-described. ${ }^{17}$

Mechanical thrombectomy (MT) in children poses unique technical challenges. The use of balloon guide catheters, as 
commonly advocated in adult MT practice, requires $8 \mathrm{Fr}$ or $9 \mathrm{Fr}$ femoral access, which is generally avoidable in children. Flow arrest or reversal, critical for clot aspiration and during mechanical retrieval through an intermediate catheter, can alternatively be achieved by a second operator maintaining aspiration on the guide catheter/sheath. ${ }^{18,19}$ In the cases of mechanical thrombectomy in this cohort, a long sheath provided means to maintain flow arrest/reversal during clot retrieval by the ADAPT (Direct Aspiration First Pass) technique. ${ }^{20}$ Additionally, the use of a long sheath in these cases also facilitated direct intermediate catheter introduction into the ICA for repeat passes, which was required in 2/3 cases, and would have also permitted to easily switch to stent retriever with the Solumbra technique. An modified thrombol$y$ sis in cerebral infarction of $2 b$ or 3 was achieved in all these cases, with one patient (162 month old, $45.5 \mathrm{~kg}$ ) requiring intra-arterial vasodilator (verapamil) for middle cerebral artery vasospasm. It is important to note that for this particular patient, vasospasm was related to the microcatheter after aspiration, and not at the long sheath tip - thus the long sheath was unlikely to be the culprit for the complication. In the one case where a long sheath was used for intra-operative angiography in a hybrid operating room, this was inserted prior to prone positioning for a cerebellar arteriovenous malformation resection. With the sheath tip positioned in the descending thoracic aorta and the proximal portion secured to the back of the patient's thigh, angiography could be performed in the prone position prior to surgical closure.

Unmistakably, none of the above described or proposed technical advantages would be meaningful if an unacceptable number of complications occurred or lack of success prevailed with the tools employed. Technical success was encountered in $92.6 \%$ of cases with the use of long sheaths for neuroendovascular procedures. The two "unsuccessful" cases were inability to achieve satisfactory ophthalmic arterial access for delivering chemotherapy in children with retinoblastoma. This was a result of individual anatomy, and can in fact be considered to be in spite of long sheath use than because of it. None of the patients in our cohort had vascular intimal injury directly attributable to the long sheath. One patient ( 2 years old) had intimal injury from the microcatheter, with focal dissection and distal thromboemboli; another (13-year old) had asymptomatic vasospasm requiring intra-arterial vasodilator therapy. Additionally, there was no increase in the rate of access-site complications. One patient (1-year old) had a groin hematoma that required a pressure dressing and pro- longed admission for observation; however, no patient in our cohort had femoro-iliac occlusions, arteriovenous fistula or pseudoaneurysm. On follow-up, there were no cases of limb ischemia. This compares well with the complication rates reported from pediatric cerebral angiography performed via small caliber short sheaths in our and other high volume centers. Hoffman et al. ${ }^{21}$, reporting on their experience with 309 consecutive cerebral angiograms, found a $2.9 \%$ rate of non-neurological complications (7 cases of bronchospasm, 1 transient femoral arterial occlusion and 1 groin hematoma) and no neurological complications. Similarly, Lin et al. ${ }^{22}$ found a 5.6\% rate of non-neurological complications and 1.1\% neurological complications in 697 consecutive procedures (429 diagnostic and 268 therapeutic).

We found no significant difference in complication rates between $\leq 15$ and $>15 \mathrm{~kg}$ groups. This is contrary to the findings reported in the literature on pediatric cardiac catheterizations. Ding et al reported arterial compromise in 11/29 (38\%) infants $\leq 15 \mathrm{~kg}$ using long sheaths, as compared to $6 / 40$ (15\%) of infants with short sheaths, used for cardiac catheterization. ${ }^{3}$ In their cohort, time to access and sheath duration were not relevant as risk factors, but weight was, with children $<5 \mathrm{~kg}$ being particularly susceptible to complications. Alexander et al. ${ }^{23}$, reporting from 486 cardiac transfemoral catheterizations in children $<18$ years, reported arterial compromise in 33 (6.8\%) children, of whom 23 (4.7\%) required treatment. Again, the smallest children, infants $<6$ months, were at an increased risk, and a femoral arterial diameter of $<3 \mathrm{~mm}$ was found to be an independent predictor for loss of arterial pulse following the procedure. Similarly, arterial occlusion following pediatric cardiac catheterization was reported by Glatz et al. ${ }^{24}$, in $4.3 \%$ of 5,715 procedures performed in children, with smaller children and larger access catheters being implicated.

Using long sheaths without short femoral sheaths, we have shown that both neurological and access-site complications can be kept within acceptable limits while performing pediatric neuroendovascular cases including in smaller children. Although our numbers are presumably too small to draw conclusions, we provide preliminary and novel data regarding the use of long sheaths in neurointerventions in children, without an increase in complications. These results however should not be misconstrued to imply that the risks of femoral arterial occlusion and neck vessel injury are insignificant. While these comparisons are a reassuring testimonial to the use of long sheaths for pediatric neuroendovascular proce- 
dures where they add definite merit, it must be pointed out that these results originate from quaternary referral centers where patients are carefully selected, procedures performed using a stringent protocols as outlined before, and neurointerventional procedures are supported by specialized pre-, intra- and post-procedural care offered by neurosurgeons, diagnostic and interventional neuroradiologists, anesthesiologists, pediatric interventional radiologists, neurointensivists, interventional radiology technologists, and nurses. This is as much attributable to strict adherence to protocol as to the availability of multi-disciplinary expertise to preempt and manage complications. Longer term data with larger numbers is required before this can be considered as routine as in adult neurointerventional practice. As such, one should still default to using the smallest access that will permit safe and complete procedure execution.

Our study had certain limitations. Firstly, this was a retrospective sample with small numbers. However, all cases were performed adhering to a standardized protocol for pre-, intra- and post-procedural periods. Although procedures performed were heterogeneous, these are indicative of the diverse etiologies of pediatric neuroendovascular procedures and retains clinical relevance for neurosurgeons and neurointerventionists involved in the care of children with these conditions. Second, we did not include long term limb and vessel outcomes, but these are unlikely to be affected following clinical follow-up during and after discharge, and with the knowledge of pediatric angiography gained over the last many decades. Third, we do not know regarding the presence and rate of asymptomatic brain and limb emboli following an intracranial procedure, and whether these are increased with the use of long sheaths. This was not the focus of this study, and would require a well-designed forward-looking study that includes dedicated diffusion weighted magnetic resonance imaging following every procedure.

\section{CONCLUSION}

Long vascular sheaths without short femoral sheaths can be safely and effectively used for pediatric neuroendovascular procedures, increasing or maintaining access ID without increasing OD. Thus, it is possible to increase the range of devices used and intracranial maneuvers that can be safely performed, thereby increasing the repertoire of the neuro- interventionist. It is crucial to maintain stringent criteria for patient selection, intra- and post-procedural protocols, as well as multi-disciplinary collaboration, in order to minimize complications.

\section{Fund}

None.

\section{Ethics Statement}

The study was approved by the Institutional Research Ethics Board (REB\# 1000056549). Written informed consent was obtained from all patients/parents for publication before the procedures. Need for individual consent for this retrospective review was waived by the REB.

\section{Conflicts of Interest}

The authors have no conflicts to disclose.

\section{Author Contributions}

Concept and design: AAD, KB, and PM. Analysis and interpretation: $A A D, W H, S B, M S$, and PM. Data collection: AAD, WH, and PM. Writing the article: $A A D, W H, K B$, and PM. Critical revision of the article: $A A D, S B, M S, P D$, and PM. Final approval of the article: $A A D, P D$, and PM. Statistical analysis: $A A D, S B$, $K B$, and $P M$. Obtained funding: $A A D$ and $S B$. Overall responsibility: $A A D, K B$, and $P M$.

\section{ORCID}

Adam A. Dmytriw: https://orcid.org/0000-0003-0131-5699

Winston Ha: https://orcid.org/0000-0001-8559-1388

Suzanne Bickford: https://orcid.org/0000-0002-5066-9322

Kartik Bhatia: https://orcid.org/0000-0002-3431-5647

Manohar Shroff: https://orcid.org/0000-0002-3791-5920

Peter Dirks: https://orcid.org/0000-0001-5718-6465

Prakash Muthusami: https://orcid.org/0000-0002-7062-9571

\section{REFERENCES}

1. Chewning R, Wyse G, Murphy K. Neurointervention for the peripheral radiologist: tips and tricks. Semin Intervent Radiol 2008;25:42-47

2. Daehnert I, Rotzsch C, Wiener M, Schneider P. Rapid right ventricular pacing is an alternative to adenosine in catheter interventional procedures for congenital heart disease. Heart 2004;90:1047-1050 
3. Ding L, Pockett C, Moore J, El-Said H. Long sheath use in femoral artery catheterizations in infants $<15 \mathrm{~kg}$ is associated with a higher thrombosis rate: proposed protocol for detection and management. Catheter Cardiovasc Interv 2016;88:1108-1112

4. Alaraj A, Wallace A, Dashti R, Patel P, Aletich V. Balloons in endovascular neurosurgery: history and current applications. Neurosurgery 2014;74 Suppl 1:S163-S190

5. Varadharajan S, Ramalingaiah AH, Saini J, Gupta AK, Devi BI, Acharya UV. Precipitating hydrophobic injectable liquid embolization of intracranial vascular shunts: initial experience and technical note. J Neurosurg 2018;129:1217-1222

6. Szajner M, Roman T, Markowicz J, Szczerbo-Trojanowska M. Onyx ${ }^{\circledR}$ in endovascular treatment of cerebral arteriovenous malformations - a review. Pol J Radiol 2013;78:35-41

7. Chapot R, Stracke P, Velasco A, Nordmeyer H, Heddier M, Stauder $\mathrm{M}$, et al. The pressure cooker technique for the treatment of brain AVMs. J Neuroradiol 2014;41:87-91

8. Ashour R, Aziz-Sultan MA, Soltanolkotabi M, Schoeneman SE, Alden TD, Hurley MC, et al. Safety and efficacy of onyx embolization for pediatric cranial and spinal vascular lesions and tumors. Neurosurgery 2012;71:773-784

9. Vollherbst DF, Sommer CM, Ulfert C, Pfaff J, Bendszus M, Möhlenbruch MA. Liquid embolic agents for endovascular embolization: evaluation of an established (Onyx) and a novel (PHIL) embolic agent in an in vitro AVM model. AJNR Am J Neuroradiol 2017;38:1377-1382

10. Gross BA, Du R. Diagnosis and treatment of vascular malformations of the brain. Curr Treat Options Neurol 2014;16:279

11. Paramasivam S, Toma N, Niimi Y, Berenstein A. Development, clinical presentation and endovascular management of congenital intracranial pial arteriovenous fistulas. J Neurointerv Surg 2013;5:184-190

12. Rosen RJ, Contractor S. The use of cyanoacrylate adhesives in the management of congenital vascular malformations. Semin Intervent Radio/ 2004;21:59-66

13. Ishikawa M, Horikawa M, Yamagami T, Uchida BT, Awai K, Kaufman JA. Embolization of arteriovenous malformations: effect of flow control and composition of n-butyl-2 cyanoacrylate and iodized oil mixtures with and without ethanol in an in vitro model. Radiology 2016;279:910-916
14. ApSimon HT, Hartley DE. Embolization of small vessels with a double-lumen microballoon catheter. Part I: design and construction. Radiology 1984;151:55-57

15. Lopera JE. Embolization in trauma: principles and techniques. Semin Intervent Radiol 2010;27:14-28

16. Kim DJ, Suh DC, Kim BM, Kim DI. Adjuvant coil assisted glue embolization of vein of galen aneurysmal malformation in pediatric patients. Neurointervention 2018;13:41-47

17. Kwon OK, Kim SH, Kwon BJ, Kang HS, Kim JH, Oh CW, et al. Endovascular treatment of wide-necked aneurysms by using two microcatheters: techniques and outcomes in 25 patients. AJNR Am J Neuroradiol 2005;26:894-900

18. Chueh JY, Kühn AL, Puri AS, Wilson SD, Wakhloo AK, Gounis MJ. Reduction in distal emboli with proximal flow control during mechanical thrombectomy: a quantitative in vitro study. Stroke 2013;44:1396-1401

19. Mokin M, Setlur Nagesh SV, Ionita CN, Levy El, Siddiqui AH. Comparison of modern stroke thrombectomy approaches using an in vitro cerebrovascular occlusion model. AJNR Am J Neuroradiol 2015;36:547-551

20. Chueh JY, Puri AS, Wakhloo AK, Gounis MJ. Risk of distal embolization with stent retriever thrombectomy and ADAPT. J Neurointerv Surg 2016;8:197-202

21. Hoffman CE, Santillan A, Rotman L, Gobin YP, Souweidane MM. Complications of cerebral angiography in children younger than 3 years of age. J Neurosurg Pediatr 2014;13:414-419

22. Lin N, Smith ER, Scott RM, Orbach DB. Safety of neuroangiography and embolization in children: complication analysis of 697 consecutive procedures in 394 patients. J Neurosurg Pediatr 2015;16:432-438

23. Alexander J, Yohannan T, Abutineh I, Agrawal V, Lloyd H, Zurakowski D, et al. Ultrasound-guided femoral arterial access in pediatric cardiac catheterizations: a prospective evaluation of the prevalence, risk factors, and mechanism for acute loss of arterial pulse. Catheter Cardiovasc Interv 2016;88:1098-1107

24. Glatz AC, Shah SS, McCarthy AL, Geisser D, Daniels K, Xie D, et al. Prevalence of and risk factors for acute occlusive arterial injury following pediatric cardiac catheterization: a large single-center cohort study. Catheter Cardiovasc Interv 2013;82:454-462 